\title{
A randomized trial of individual versus group- format exercise and self-management in individuals with Parkinson's disease and comorbid depression
}

\author{
This article was published in the following Dove Press journal: \\ Patient Preference and Adherence \\ 19 May 2017 \\ Number of times this article has been viewed
}

\author{
Martha Sajatovic ${ }^{1,2}$ \\ Angela L Ridgel ${ }^{3}$ \\ Ellen MWalter ${ }^{1,4}$ \\ Curtis M Tatsuoka ${ }^{1,2}$ \\ Kari Colón-Zimmermann ${ }^{2}$ \\ Riane K Ramsey ${ }^{2}$ \\ Elisabeth Welter ${ }^{2}$ \\ Steven A Gunzler ${ }^{1,4}$ \\ Christina MWhitney ${ }^{1,4}$ \\ Benjamin L Walter ${ }^{1,4}$ \\ 'Department of Neurology, Case \\ Western Reserve University \\ School of Medicine, ${ }^{2}$ Neurological \\ and Behavioral Outcomes Center, \\ University Hospitals Cleveland \\ Medical Center, Cleveland, \\ ${ }^{3}$ Department of Exercise Physiology, \\ Kent State University, Kent, \\ ${ }^{4}$ Movement Disorders Center, \\ University Hospitals Cleveland \\ Medical Center, Cleveland, OH, USA
}

Background: Depression is common in people with Parkinson's disease (PD), and exercise is known to improve depression and PD. However, lack of motivation and low self-efficacy can make exercise difficult for people with PD and comorbid depression (PD-Dep). A combined group exercise and chronic disease self-management (CDSM) program may improve the likelihood that individuals will engage in exercise and will show a reduction in depression symptoms. The purpose of this study was to compare changes in depression in PD-Dep between individual versus group exercise plus CDSM and to examine participant adherence and perception of the interventions.

Methods: Participants $(\mathrm{N}=30)$ were randomized to either Enhanced EXerCisE thErapy for PD (EXCEED; group CDSM and exercise) or self-guided CDSM plus exercise. Outcomes were change in depression assessed with the Montgomery-Asberg Depression Rating Scale (MADRS), cognition, apathy, anxiety, sleep, quality of life, motor function, self-efficacy, and patient satisfaction.

Results: Both groups showed significant improvement in MADRS $(P<0.001)$ with no significant group difference. Individuals in EXCEED group enjoyed the group dynamics but noted difficulty with the fixed-time sessions.

Conclusion: Both group CDSM plus exercise and self-guided CDSM plus exercise can improve depression in PD-Dep. These findings suggest that development of a remotely delivered groupbased CDSM format plus manualized exercise program could be useful for this population.

Keywords: community-based research, mental health, rehabilitation, physical activity

\section{Introduction}

By 2030, the population over age 60 will have grown 3.5 times as rapidly as the total population. ${ }^{1}$ Parkinson's disease (PD), an aging-related neurological disorder, is expected to double in prevalence by $2040 .^{2}$ In the US alone, economic impact of PD, including treatment, social security payments, and lost employment income, exceeded $\$ 14.4$ billion in $2010 .^{2}$ Although PD is identified as a movement disorder, non-motor symptoms such as depression and cognitive deficits negatively affect quality of life and independence. ${ }^{3-6}$ Approximately 1 in 2 people with PD will develop comorbid depression (PD-Dep). ${ }^{7-9}$ Depression has been found to be an independent predictor of poor quality of life in individuals with $\mathrm{PD} .^{10}$

Optimal treatments for PD-Dep have not been clearly established. ${ }^{11}$ Antidepressant drug therapies have inconsistent benefits for PD-Dep ${ }^{12}$ and may cause burdensome side effects. Serotonin reuptake inhibitors can worsen PD symptoms, ${ }^{13}$ while tricyclic antidepressants can cause orthostatic hypotension, sedation, cardiac arrhythmias,
Correspondence: Angela L Ridgel Department of Exercise Physiology, Kent State University, 350 Midway

Drive, Kent, OH 44242, USA

$\mathrm{Tel}+\mathrm{I} 3306727495$

Fax +I 3306722250

Email aridgel@kent.edu 
and anticholinergic effects. ${ }^{14}$ Cognitive behavioral therapy (CBT) has been demonstrated to help some individuals with PD-Dep, but it may not be accepted by some older patients and lengthy CBT formats limit generalizability. ${ }^{15-19}$ Cognitive deficits in PD may also impede engagement in psychotherapies that rely on psychological "homework".

Both aerobic and resistance exercise have been shown to be helpful for PD and for depression, independently., ${ }^{9,20,21}$ Although the mechanisms for these exercise-induced improvements are not known, aerobic exercise has been shown to increase the levels of brain-derived neurotrophic factor (BDNF). ${ }^{22}$ Serum levels of BDNF in those with PD-Dep are generally lower than that of healthy controls. ${ }^{23,24} \mathrm{~A}$ recent meta-analysis suggested that, although the current literature does not show strong support for exercise-based improvements in PD-Dep, further investigation is warranted. ${ }^{25}$ However, reduced motivation, apathy, anxiety, low self-efficacy, fear of falling, and excessive sleepiness in people with PD and depression may be a significant barrier to engagement in exercise programs. ${ }^{26-28}$ Therefore, there is a need to develop interventions that encourage and support adherence to exercise behaviors in this population.

Chronic disease self-management (CDSM) can improve the health and quality of life of people with multiple morbidities, including depression. ${ }^{29-33}$ CDSMs are defined as behavioral interventions that reduce the negative consequences of chronic health conditions through medical management, maintenance or creation of new meaningful behaviors or life roles, and management of emotional reactions. ${ }^{30}$ While specific elements of CDSM may vary, group education sessions $^{33}$ and health coaching are 2 formats in which selfmanagement among those with chronic health conditions can be implemented. ${ }^{34}$ As an example, CDSM programs have been proven successful in reducing diabetes symptoms and hypertension in older adults. ${ }^{32}$ However, the authors are not aware of any studies that have examined the benefits of group CDSM programs plus group exercise in individuals with PD-Dep.

Given the evidence base for CDSM, it seems reasonable that adding CDSM to an exercise approach for people with PD-Dep may help overcome barriers to exercise. To inform the development of an approach that is acceptable and practical for patients with PD-Dep, this study compared 2 experimental interventions that combined exercise and CDSM. The interventions were Enhanced EXerCisE thErapy for PD (EXCEED) versus self-guided exercise and self-management (SGE). EXCEED used individuals with PD-Dep to help deliver the intervention in a group format (peer-led), while SGE relied on detailed written materials, which people with PD-Dep accessed and used at their own pace. The primary aim of this study was to determine how each of these interventions altered depression and to determine participant acceptance and adherence to the interventions. An exploratory aim was to determine if factors of neural health and inflammation were altered after these interventions. While there were common elements to both interventions (exercise + CDSM), it was hypothesized that EXCEED, because of the more intensive social support and group format, ${ }^{35,36}$ would be associated with greater improvement in depression symptoms and greater adherence to the program than SGE.

\section{Methods}

EXCEED and SGE were assessed via a prospective, 6-month, randomized controlled trial in 30 patients with PD-Dep. At baseline, individuals were randomized (Figure 1; $1: 1$ ratio) to either EXCEED $(n=15)$ or $\operatorname{SGE}(n=15)$. The primary outcome was change in depression symptoms from baseline. Secondary outcomes included cognition, apathy, anxiety, sleep, quality of life, motor function, self-efficacy, and patient satisfaction. As an exploratory outcome related to possible mechanisms for changes in depression, selected biomarkers of inflammation (interleukin 6 [IL-6], tumor necrosis factor alpha [TNF-alpha]) and neuronal integrity (BDNF) were also examined.

\section{Participants}

Inclusion criteria were the following: 1) a diagnosis of idiopathic PD with Hoehn and Yahr stage $\leq \mathrm{III} ;{ }^{37}$ 2) a diagnosis of unipolar major depression with a Montgomery-Asberg Depression Rating Scale (MADRS) ${ }^{38}$ score $\geq 14$; 3 ) the ability to walk independently; and 4) on a stable dose of PD medication for $\geq 2$ weeks and antidepressant medication (if applicable) for $\geq 4$ weeks prior to enrollment. The study was approved by the University Hospitals of Cleveland Institutional Review Board, and written informed consent was obtained from all the participants who were recruited from local medical practices and through community outreach. Medical clearance for exercise was obtained from primary clinicians. Exclusion criteria were 1) Mini-Mental State Examination $(\mathrm{MMSE})^{39}$ score of $\left.<24,2\right)$ unstable cardiovascular disease, 3) high fall risk, or 4) other uncontrolled chronic conditions that would interfere with the safety and conduct of the protocol or interpretation of the results.

\section{Interventions}

Adapting successful curricula for central nervous system disorders, ${ }^{40-42}$ a CDSM for PD-Dep was developed. Exercise 


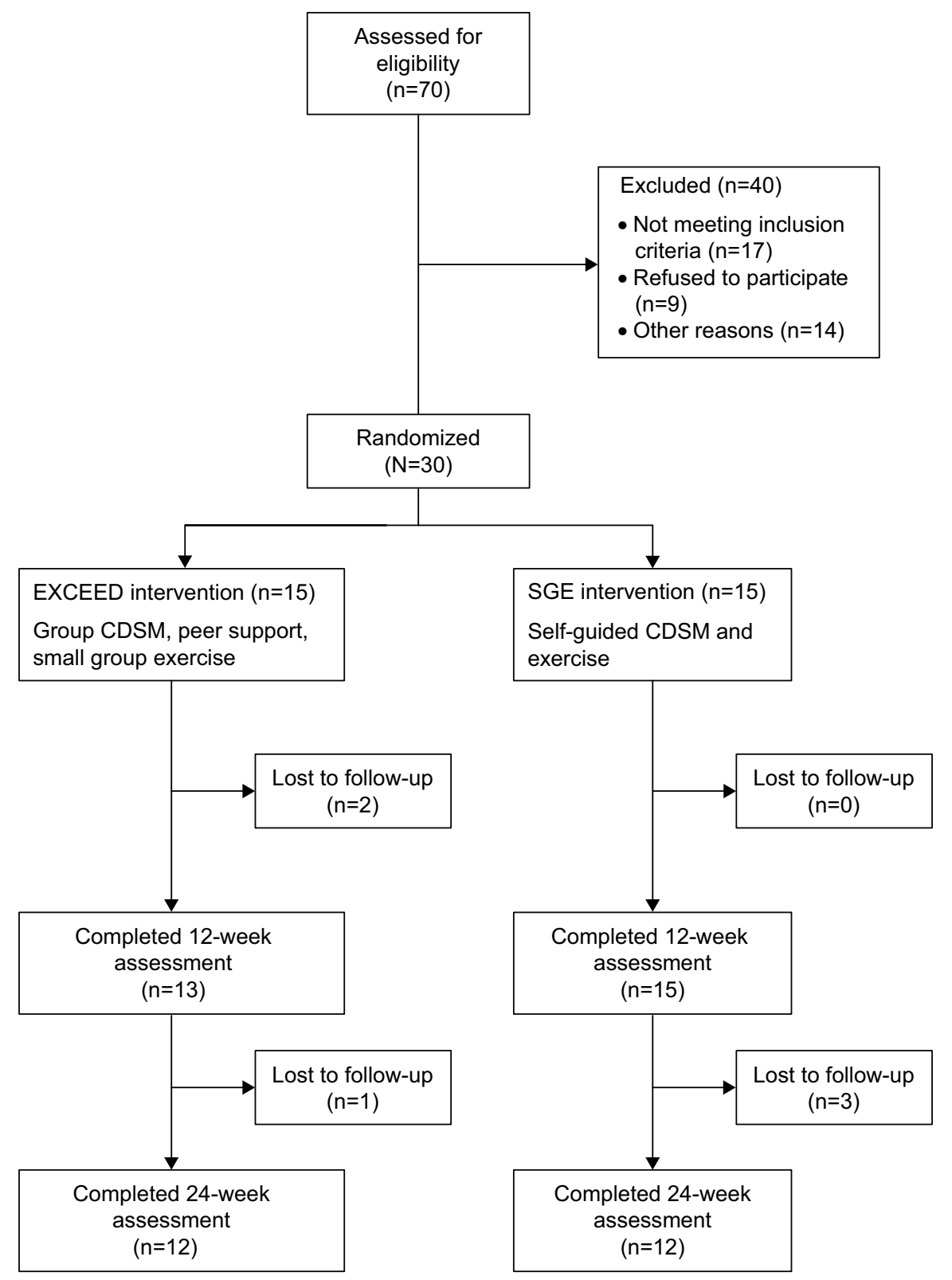

Figure I Consort diagram.

Abbreviations: EXCEED, Enhanced EXerCisE thErapy for Parkinson's Disease; CDSM, chronic disease self-management; SGE, self-guided exercise.

was adapted from a successful program developed for $\mathrm{PD}^{43}$ and consisted of 3 times/week sessions conducted at a local exercise facility. EXCEED included weekly group CDSM sessions with peer support and guided group exercise. The CDSM sessions were manualized, and emphasized problem identification and goal setting. The 1-hour-long group sessions with 7-8 PD-Dep patients were co-lead by a nurse educator with expertise in PD and a trained peer educator with PD-Dep. Detailed instruction manuals for the exercise program helped assure consistency, and exercise sessions were led by a certified personal trainer. ${ }^{44}$ Exercise consisted of fast-paced, low-resistance cycling for 20 minutes followed by strength training for 20 minutes using a progressive sequence of resistance bands. After 12 weeks, individuals continued to exercise on their own, using either weight machines or resistance bands.

SGE participants had a single initial in-person group orientation to the same fitness facility followed by flexible and free access. Participants received detailed written instructions for the exercise program and access to the fitness instructor, as needed. Participants were asked to exercise at least 3 times per week and received weekly phone calls during the first 12 weeks to self-report on their exercise. In addition, they received the same CDSM information and were instructed to read and practice the CDSM materials at their own pace. 


\section{Outcome measures}

Participants were assessed at baseline, and 12 and 24 weeks. Depression, the primary outcome, was measured with the MADRS. To minimize rater bias, administration of the MADRS was video-recorded and scored by blinded raters. Secondary assessments measured general cognition (Montreal Cognitive Assessment, MoCA),${ }^{45}$ apathy (Apathy Scale),${ }^{46}$ anxiety (Covi Anxiety Scale), ${ }^{47}$ self-efficacy (General SelfEfficacy Scale), sleep (Scales for Outcomes in PD - Sleep, SCOPA-Sleep), ${ }^{48}$ and motor symptoms (Movement Disorders Society-Unified Parkinson's Disease Rating Scale-III, MDS-UPDRS-III). ${ }^{49}$ MDS-UPDRS-III was assessed in the "ON" state within 2 hours after a dose of PD medication, was video-recorded, and scored by blinded raters.

Participant satisfaction was evaluated at weeks 12 and 24. Individuals in both groups rated the usefulness, comprehensiveness, perceived burden, and relevance of their assigned intervention along with patient satisfaction with timing, length, and number of sessions using a custom survey (Table 1).

This study also tested for markers of neuroprotection (BDNF) and inflammation (TNF-alpha, IL-6) at baseline, and at 12 and 24 weeks, as these can be affected by exercise and mood. ${ }^{24,50,51}$ BDNF is proposed as a mechanistic biomarker for PD-Dep because its levels are generally lower in those with PD-Dep than that of healthy controls, ${ }^{23,24}$ and exercise has been shown to increase the levels of BDNF. ${ }^{22}$ Blood samples were placed on ice and centrifuged within 30 minutes. Two aliquots were taken from the middle third of the plasma, and stored at $-70^{\circ} \mathrm{C}$. Plasma samples were assayed using commercial enzyme-linked immunosorbent assay (ELISA) per manufacturer instructions (Quantikine ${ }^{\circledR}$ ELISA Human BDNF Immunoassay; R\&D Systems, Minneapolis, MN, USA).
Elevated levels of cytokines are present in PD suggesting that inflammation may play a role in the pathophysiology of the disease. ${ }^{52,53}$ However, it has been suggested that the increase in IL-6 after moderate-intensity exercise promotes an anti-inflammatory response..$^{54,55}$ In contrast, pro-inflammatory cytokines, like TNF-alpha, are reduced after moderate-intensity exercise. For TNF-alpha and IL-6, blood was allowed to clot for 30 minutes, and then centrifuged. Serum aliquots were stored at $-70^{\circ} \mathrm{C}$. Serum samples were assayed using commercial kits per manufacturer instructions (Quantikine ${ }^{\circledR}$ HS ELISA Human IL-6 Immunoassay; R\&D Systems).

\section{Sample size analysis}

A priori sample size analysis was informed by MADRS change from baseline results from a previous self-management study conducted by these investigators. ${ }^{41}$ For the primary depression outcome, a mean change of 12.08 points (standard deviation $[\mathrm{SD}]=7.01$ ) on the MADRS was considered for those in the EXCEED arm. Moreover, we assume that the respective mean change for those in the control arm is 4.0, which is a large projected gain for a control. For a 2-sided test with alpha $=0.05$, and 15 subjects per arm, power will be $>0.80$ when the total sample size is 26 . Attrition is estimated to be at most $10 \%$ based on the initial reaction to a preliminary implementation. Hence, a total sample of 30 subjects, even with expected attrition, should provide power $>0.80$.

\section{Data analysis}

Descriptive data analysis was performed using standard univariate techniques. Since types and formulations of PD drugs differed, L-dopa medication equivalents were standardized. ${ }^{56}$

Table I Custom survey to evaluate participant satisfaction with EXCEED

\begin{tabular}{ll}
\hline Section I & $\begin{array}{c}\text { Strongly Agree Neutral/do Disagree } \begin{array}{c}\text { Strongly } \\
\text { agree }\end{array} \\
\text { disagree }\end{array}$ \\
\hline EXCEED is useful & \\
EXCEED covers all/most of the important issues & Too long \\
EXCEED addresses the issues that are important to my particular situation & Just \\
The benefit of EXCEED exceeds the burden or hassle of attending EXCEED sessions & \\
\hline Section $\mathbf{2}$ & \\
\hline The number of sessions & \\
EXCEED timing (period of time it took to complete all sessions) & \\
The length of time for each EXCEED session & \\
\hline Section $\mathbf{3}$ & \\
\hline What did you like best about EXCEED? & \\
What did you like least about EXCEED? & \\
What were the biggest problems (if any) in how the EXCEED sessions took place? & \\
How can we improve EXCEED? & \\
\hline
\end{tabular}

Abbreviation: EXCEED, Enhanced EXerCisE thErapy for Parkinson's Disease. 
Categorical outcomes were compared by either chi-square or Fisher's exact test. Continuous outcomes were compared via Student's $t$-test. Data analysis of all outcomes compared baseline values to 12- and 24-week outcomes using SPSS software v23. Type I error levels were set at 0.05 .

\section{Results}

Table 2 shows the baseline demographic and clinical characteristics of the participants. Mean age of the participants was 70 years. The majority were Caucasian men (63\%). Participants were moderately depressed, with a mean MADRS score of 20.8. On average, onset of the first depressive episode was at least a decade prior to study participation, and 2 individuals had a history of suicide attempt. At baseline, 18 (60\%) of 30 individuals were on antidepressants. Cognitive impairment was generally mild to moderate with a mean MMSE score at screening of 27.9 and an MoCA score at baseline of 22.9. Individuals had PD for approximately 7 years at baseline, and all used PD medication daily. Medical comorbidity was extensive, as noted by the self-reported Charlson Comorbidity Index. ${ }^{57}$

\section{EXCEED versus SGE group comparisons}

The EXCEED and SGE groups had similar baseline demographic and clinical profiles, although the EXCEED group had PD for a slightly longer duration (7.3 years vs 6.4 years), were receiving higher doses of PD treatment, and had more extensive medical comorbidity (Table 2). Education and L-dopa dosage equivalent were significantly lower in the
SGE group $(P<0.05)$. Longitudinal mood outcomes were similar between the groups. L-dopa equivalents received by individuals in EXCEED versus SGE group showed no significant changes. Antidepressant medication changes were modest and also similar between the groups. Four individuals had increases or additions to antidepressant medication before week 12 of the study ( 3 in SGE and 1 in EXCEED group). Two individuals had increases or additions to antidepressant medications after week 12 of the study (both in EXCEED group), and 1 individual had a reduction in antidepressant dose after week 12 of the study (EXCEED group).

Both groups attended a similar number of exercise sessions (EXCEED: $M=20.7, \mathrm{SD}=8.1$; SGE: $M=22, \mathrm{SD}=8.0$ ). Three individuals dropped out of EXCEED, two due to unrelated health concerns and 1 due to schedule constraints (Figure 1). One individual dropped out of SGE after week 11 due to arthritis pain, and 1 individual in SGE group was diagnosed with dementia and only completed a portion of the week 24 assessments. One participant in SGE group had a recurrence of lower back pain within 2 weeks of starting the exercise program. This individual did not continue the exercise, but completed the 12- and 24-week assessments.

The EXCEED participants were very satisfied with the social aspects of group attendance. They cited this as a major beneficial aspect of their program, and some of the participants continued to interact after study completion. Participants in SGE group found it relatively easy to fit exercise into their lives, while attending the fixed-time groups was challenging for some EXCEED

Table 2 Demographic and clinical characteristics of the overall sample and by intervention group at baseline

\begin{tabular}{|c|c|c|c|}
\hline Variable & Total $(\mathbf{N}=\mathbf{3 0})$ & EXCEED $(\mathrm{N}=15)$ & SGE $(N=15)$ \\
\hline Age (years), mean (SD, range) & $70(7.9,53-83)$ & $69.8(9.3)$ & $70.3(6.5)$ \\
\hline Education, mean number of years (SD) & $14.6(2.8)$ & $16.0(3.1)$ & $13.3(1.6)^{b}$ \\
\hline \multicolumn{4}{|l|}{ Gender } \\
\hline Female, N (\%) & II (36.7) & $4(26.7)$ & $7(46.7)$ \\
\hline Male, N (\%) & $19(63.3)$ & II (73.3) & $8(53.3)$ \\
\hline \multicolumn{4}{|l|}{ Ethnicity } \\
\hline Caucasian, N (\%) & $29(96.7)$ & $14(93.3)$ & $15(100.0)$ \\
\hline African American, N (\%) & I (3.3) & I (6.7) & $0(0.0)$ \\
\hline Years since PD diagnosis, mean (SD) & $6.8(5.3)$ & $7.3(3.4)$ & $6.4(6.7)$ \\
\hline Years since onset of depression, mean (SD) & $13.0(19.1)$ & $13.9(18.3)$ & I2.I (20.5) \\
\hline Antidepressant use, N (\%) & $18(60.0)$ & $7(46.7)$ & II (73.3) \\
\hline Total LDEa, mean (SD) & $717.8(394.2)$ & $934.6(434.5)$ & $529.9(237.3)^{\mathrm{b}}$ \\
\hline $\mathrm{CCl}$, mean $(\mathrm{SD})$ & $2.6(2.6)$ & $3.4(3.2)$ & $1.7(1.6)$ \\
\hline MMSE, mean (SD) & $27.9(2.0)$ & $28.2(1.9)$ & $27.6(2.2)$ \\
\hline MADRS, mean (SD) & $20.8(6.5)$ & $20.9(5.3)$ & $21.5(6.1)$ \\
\hline MoCA, mean (SD) & $22.9(4.3)$ & $22.6(4.8)$ & $23.2(4.0)$ \\
\hline
\end{tabular}

Notes: ${ }^{2} \mathrm{LDE}$ based on $\mathrm{N}=28$. ${ }^{\mathrm{P}} \mathrm{P}<0.05$.

Abbreviations: EXCEED, Enhanced EXerCisE thErapy for Parkinson's Disease; SGE, self-guided chronic disease self-management plus exercise; SD, standard deviation; PD, Parkinson's disease; LDE, L-dopa dosage equivalent; CCI, Charlson Comorbidity Index; MMSE, Mini-Mental State Examination; MADRS, Montgomery-Asberg Depression Rating Scale; MoCA, Montreal Cognitive Assessment. 
participants. While no individuals in the EXCEED group attended all 12 sessions, 6 individuals attended either 10 or 11 sessions. Overall, both EXCEED ( $n=13 / 13,100 \%)$ and SGE $(n=11 / 13,84.6 \%)$ participants agreed or strongly agreed that they found participation in the program to be useful. Similarly, groups found the benefit to be worth the burden of participating. Major criticisms for both groups were transportation and logistic difficulties in getting to the fitness center.

\section{Combined group therapeutic effects}

As there were no outcome differences between the groups, the combined sample was then analyzed, which showed significant improvements in depression. Table 3 shows that the combined group (EXCEED + SGE) had significantly improved MADRS at 12-week follow-up. At 24-week follow-up, the group as a whole maintained improvements in MADRS (24 weeks: $M=14.2, \mathrm{SD}=8.5, t(25)=4.87$, $P<0.001)$. Antidepressant drug use was relatively similar over the course of the trial. PD drug L-dopa equivalents showed no significant change $(t(24)=-1.54, P=0.136)$ from baseline $(M=702.1, \mathrm{SD}=352.0)$ to week $24(M=754.5$, $\mathrm{SD}=432.8$ ). There were no significant changes in apathy, anxiety, or self-efficacy, as noted in Table 3. Night sleep quality on the SCOPA was slightly worse $(P=0.03)$ at 24 weeks. In addition, global cognition as measured by the MoCA was modestly better for the combined data at 24 weeks $(M=25.2$, $\mathrm{SD}=5.1, t(25)=-2.56, P=0.017)$. There was no significant change in MDS-UPDRS-III over the 24 weeks.
There was a significant increase in plasma BDNF that corresponded to the initial 12-week "intensive" portion of the interventions (weekly groups for EXCEED and weekly check-in calls for SGE). At the 24-week follow-up, plasma BDNF was still significantly greater than baseline, but values decreased when compared to the 12-week data. There was a minimal change in IL-6 and in TNF-alpha. At baseline, $14(46.7 \%)$ of 30 individuals had falls in the past 3 months. The incidence of falls decreased slightly, with 9 (30\%) of 30 individuals reporting falls between baseline and 12 -week follow-up and between 12-week and 24-week follow-up.

\section{Discussion}

In spite of the fact that depression is common in PD, worsens outcomes, and is the main contributor to poor health in $\mathrm{PD},{ }^{58}$ there are few effective therapies for PD-Dep. ${ }^{11}$ In this prospective trial that combined 2 evidence-based approaches, exercise and CDSM elements, depressive outcomes improved. This study found no evidence that a group program was superior to an individualized program, although the social elements of the group format appeared important to patients with PD-Dep. Allowing individuals to exercise at their own pace using a manualized curriculum appeared practical and highly acceptable. While this study has important methodological limitations, findings suggest some clinically relevant interpretations.

Consistent with the initial hypothesis, individuals with PD-Dep who participated in exercise plus CDSM had improvement in depression. Though depression in PD may be

Table 3 Changes in primary and secondary outcomes at 12 and 24 weeks for EXCEED and SGE groups combined

\begin{tabular}{|c|c|c|c|c|c|}
\hline Variable & $\begin{array}{l}\text { Baseline } \\
\text { mean (SD) }\end{array}$ & $\begin{array}{l}\text { Week I2 } \\
\text { mean (SD) }\end{array}$ & $\begin{array}{l}\text { Week } 12 \\
\text { difference }(P)\end{array}$ & $\begin{array}{l}\text { Week } 24 \\
\text { mean (SD) }\end{array}$ & $\begin{array}{l}\text { Week } 24 \\
\text { difference }(P)\end{array}$ \\
\hline MADRS $(n=26)$ & $21.2(6.3)$ & $15.2(8.0)$ & $-6.0(<0.001)$ & I4.2(8.5) & $-6.9(<0.001)$ \\
\hline $\operatorname{MoCA}(n=26)$ & $23.3(4.1)$ & $25.2(3.7)$ & $1.9(0.002)$ & $25.2(5.1)$ & $1.9(0.017)$ \\
\hline Covi Anxiety $(n=25)$ & $4.2(1.6)$ & $5.0(2.5)$ & $0.8(0.089)$ & $4.4(2.0)$ & $0.2(0.587)$ \\
\hline Apathy Scale $(n=25)$ & $16.8(4.0)$ & $17.0(3.8)$ & $0.2(0.662)$ & $16.9(4.3)$ & $0.1(0.870)$ \\
\hline GSE Scale $(n=25)$ & $29.2(4.2)$ & $28.7(3.1)$ & $-0.5(0.487)$ & $29.2(4.2)$ & $0.0(0.953)$ \\
\hline MDS-UPDRS-III (n=25) & $33.8(9.1)$ & $32.5(8.2)$ & $-1.3(0.395)$ & $33.1(8.8)$ & $-0.7(0.730)$ \\
\hline \multicolumn{6}{|l|}{ SCOPA-Sleep $(n=25)$} \\
\hline Night sleep & $10.4(3.2)$ & $9.8(3.4)$ & $-0.6(0.4 \mid 0)$ & $12.1(4.1)$ & $1.7(0.030)$ \\
\hline Daytime sleepiness & $12.0(3.3)$ & $11.3(3.3)$ & $-0.7(0.133)$ & II.4 (3.2) & $-0.6(0.403)$ \\
\hline Overall sleep & $3.4(1.4)$ & $3.2(1.5)$ & $-0.2(0.657)$ & $3.0(1.9)$ & $-0.4(0.205)$ \\
\hline \multicolumn{6}{|l|}{ Biomarkers (pg/mL) } \\
\hline IL-6 (n=25) & $1.9(1.0)$ & $1.7(1.0)$ & $-0.2(0.998)$ & $1.8(1.1)$ & $-0.1(0.253)$ \\
\hline TNF-alpha $(n=25)$ & I.3 (0.4) & $1.4(0.5)$ & $0.1(0.419)$ & $\mathrm{I} .4(0.4)$ & $0.1(0.980)$ \\
\hline BDNF $(n=25)$ & $26.8(15.6)$ & $90.0(166.4)$ & $63.2(<0.001)$ & $38.5(46.2)$ & I I.7 (0.043) \\
\hline
\end{tabular}

Note: Significant differences are highlighted in bold.

Abbreviations: EXCEED, Enhanced EXerCisE thErapy for Parkinson's Disease; SGE, self-guided chronic disease self-management plus exercise; SD, standard deviation; MADRS, Montgomery-Asberg Depression Rating Scale; MoCA, Montreal Cognitive Assessment; GSE, General Self-Efficacy; MDS-UPDRS-III, Movement Disorders SocietyUnified Parkinson's Disease Rating Scale-III; SCOPA-Sleep, Scales for Outcomes in Parkinson's Disease - Sleep; IL-6, interleukin 6; TNF-alpha, tumor necrosis factor alpha; BDNF, brain-derived neurotrophic factor. 
related to the psychological distress of having a progressive neurological disease, there is growing evidence for a possible neurobiological link between depression and PD. Depression appears to be relatively common among individuals who later develop $\mathrm{PD}^{59}$ and may represent a prodromal non-motor symptom of PD. Given strong clinical relevance of a care approach that can improve PD-Dep, a consideration for future work might include using exercise + CDSM in newly diagnosed individuals with PD.

In contrast to the original expectations, the authors did not find that group format leads to superior outcomes in PD-Dep. It is possible that individuals who agree to participate in a research study may be more motivated to participate in both exercise and CDSM than the general population of people with PD-Dep, which could explain why the SGE participants did so well. Alternatively, being able to exercise at times that are most convenient may be an advantage for individuals with PD, who may have driving or other transportation limitations. The findings also suggest that group input and peer education provide helpful and important social support. A possible real-world model of care that is informed by the findings might involve individuals with PD-Dep being able to access fitness centers in their community (using the manualized SGE format) while participating in CDSM. This format is being investigated by the study team.

Patients with PD are at a greater risk of dementia compared to the general population, and the presence of mild cognitive impairment early in the course of PD predicts a highly increased risk of dementia. ${ }^{60}$ In this trial, a modest improvement in cognition in both EXCEED and SGE participants was observed over the course of 24 weeks, although specific cognitive assessments did not show improvement. It is possible that change in cognition could have been accounted for by improvement in depression severity. Given the paucity of interventions that are known to specially improve cognition in $\mathrm{PD},{ }^{61}$ future work on this topic in patients with PD-Dep is needed.

This study did show a significant increase in plasma BDNF after the intervention. Plasma BDNF levels have been shown to correlate with PD disease duration and motor performance. ${ }^{62}$ The findings are consistent with previous literature which show that exercise increases the levels of BDNF and contributes to brain health..$^{22,63,64}$ This suggests that changes in the levels of BDNF may contribute to improvements in depression symptoms and global cognition. Although it was anticipated that markers of inflammation (IL-6 and TNF-alpha) would decrease, there was no change after the exercise intervention.

Limitations of this study include small sample size, relatively short duration, single-site location, low representation of minorities, and the fact that this study did not compare exercise alone versus CDSM alone. These findings may not generalize to PD patients with more advanced disease. The randomized design and use of raters blind to randomization assignment are strengths that minimize some of these limitations. Future studies should include a true control arm and a more ethnically diverse sample. In conclusion, a care approach that combines exercise plus CDSM elements may be associated with reduced depressive symptoms in people with PD-Dep. Further studies are needed to investigate how evidence-based approaches to manage depression in people with aging-related neurological conditions like PD might be implemented in real-world or community settings.

\section{Acknowledgments}

$\mathrm{V}$ Forro assisted with data collection and project management. R Phillips, K Wilson, and F Peters assisted with fitness assessment. K Smyth provided insight into the design of the project. The authors also thank the staff of LifeWorks of Southwest General, Middleburg Heights, $\mathrm{OH}$, for assisting with fitness training and program implementation. This study was supported by the Case Western Reserve Spitz Brain Health Innovation Fund. Support for this project was also provided by the Clinical and Translational Science Collaborative (Dahms Clinical Research Unit) NIH grant number UL1 RR024989, and by the PD advocacy group, Courageous Steps.

\section{Disclosure}

MS has research grants from Pfizer, Merck, Alkermes, Janssen, Reuter Foundation, Woodruff Foundation, Reinberger Foundation, National Institute of Health (NIH), and Centers for Disease Control (CDC). She is a consultant to Bracket, Otsuka, Pfizer, Neurocrine, and Supernus, and has received royalties from Springer Press, Johns Hopkins University Press, Oxford Press, UpToDate, and Lexicomp. EM Walter is a consultant to US WorldMeds and Teva. BLW has research grants from NIH/NINDS, Teva Pharmaceuticals, UCB, Inc., Medtronic, and US WorldMeds. He is a consultant to Teva and Medtronic and is on the speaker's bureau for Teva, Medtronic, US WorldMeds, and UCB, Inc. The other authors report no conflicts of interest in this work.

\section{References}

1. Schenkman M, Hall DA, Baron AE, Schwartz RS, Mettler P, Kohrt WM Exercise for people in early- or mid-stage Parkinson disease: a 16-month randomized controlled trial. Phys Ther. 2012;92(11):1395-1410.

2. Kowal SL, Dall TM, Chakrabarti R, Storm MV, Jain A. The current and projected economic burden of Parkinson's disease in the United States. Mov Disord. 2013;28(3):311-318. 
3. Jankovic J. Parkinson's disease: clinical features and diagnosis. J Neurol Neurosurg Psychiatry. 2008;79(4):368-376.

4. Munneke M, Nijkrake MJ, Keus SH, et al. Efficacy of communitybased physiotherapy networks for patients with Parkinson's disease: a cluster-randomised trial. Lancet Neurol. 2010;9(1):46-54.

5. Yang S, Sajatovic M, Walter BL. Psychosocial interventions for depression and anxiety in Parkinson's disease. J Geriatr Psychiatry Neurol. 2012;25(2):113-121.

6. Fisher $\mathrm{BE}, \mathrm{Wu} \mathrm{AD}$, Salem GJ, et al. The effect of exercise training in improving motor performance and corticomotor excitability in people with early Parkinson's disease. Arch Phys Med Rehabil. 2008;89(7): $1221-1229$

7. Aarsland D, Emre M, Lees A, Poewe W, Ballard C. Practice parameter: evaluation and treatment of depression, psychosis, and dementia in Parkinson disease (an evidence-based review): report of the Quality Standards Subcommittee of the American Academy of Neurology. Neurology. 2007;68(1):80; author reply 81 .

8. DiMatteo MR, Lepper HS, Croghan TW. Depression is a risk factor for noncompliance with medical treatment: meta-analysis of the effects of anxiety and depression on patient adherence. Arch Intern Med. 2000; 160(14):2101-2107.

9. Blumenthal JA, Smith PJ, Hoffman BM. Is exercise a viable treatment for depression? ACSMs Health Fit J. 2012;16(4):14-21.

10. Forsaa EB, Larsen JP, Wentzel-Larsen T, Herlofson K, Alves G. Predictors and course of health-related quality of life in Parkinson's disease. Mov Disord. 2008;23(10):1420-1427.

11. Kim JS, Oh YS, Lee KS, Kim YI, Yang DW, Goldstein DS. Association of cognitive dysfunction with neurocirculatory abnormalities in early Parkinson disease. Neurology. 2012;79(13):1323-1331.

12. Martinez-Horta S, Pagonabarraga J, Fernandez de Bobadilla R, Garcia-Sanchez C, Kulisevsky J. Apathy in Parkinson's disease: more than just executive dysfunction. J Int Neuropsychol Soc. 2013;19(5): 571-582.

13. Calderwood MS, Kleinman K, Bratzler DW, et al. Use of Medicare claims to identify US hospitals with a high rate of surgical site infection after hip arthroplasty. Infect Control Hosp Epidemiol. 2013;34(1): 31-39.

14. Senek M, Nyholm D. Continuous drug delivery in Parkinson's disease. CNS Drugs. 2014;28(1):19-27.

15. Muller T, Gerlach M, Youdim MB, Riederer P. Psychiatric, nonmotor aspects of Parkinson's disease. Handb Clin Neurol. 2012;106: $477-490$.

16. Anderson KE. Dementia in Parkinson's disease. Curr Treat Options Neurol. 2004;6(3):201-207.

17. Dubois B, Burn D, Goetz C, et al. Diagnostic procedures for Parkinson's disease dementia: recommendations from the movement disorder society task force. Mov Disord. 2007;22(16):2314-2324.

18. Emre M, Aarsland D, Brown R, et al. Clinical diagnostic criteria for dementia associated with Parkinson's disease. Mov Disord. 2007;22(12): 1689-1707; quiz 1837.

19. Janvin CC, Aarsland D, Larsen JP. Cognitive predictors of dementia in Parkinson's disease: a community-based, 4-year longitudinal study. J Geriatr Psychiatry Neurol. 2005;18(3):149-154.

20. Cooney GM, Dwan K, Greig CA, et al. Exercise for depression. Cochrane Database Syst Rev. 2013;9:CD004366.

21. Richard IH, McDermott MP, Kurlan R, et al. A randomized, doubleblind, placebo-controlled trial of antidepressants in Parkinson disease. Neurology. 2012;78(16):1229-1236.

22. Knaepen K, Goekint M, Heyman EM, Meeusen R. Neuroplasticity exercise-induced response of peripheral brain-derived neurotrophic factor: a systematic review of experimental studies in human subjects. Sports Med. 2010;40(9):765-801.

23. Molendijk ML, Spinhoven P, Polak M, Bus BA, Penninx BW, Elzinga BM. Serum BDNF concentrations as peripheral manifestations of depression: evidence from a systematic review and meta-analyses on 179 associations (N=9484). Mol Psychiatry. 2014;19(7):791-800.
24. Ricci V, Pomponi M, Martinotti G, et al. Antidepressant treatment restores brain-derived neurotrophic factor serum levels and ameliorates motor function in Parkinson disease patients. J Clin Psychopharmacol. 2010;30(6):751-753.

25. Lauze M, Daneault JF, Duval C. The effects of physical activity in Parkinson's disease: a review. J Parkinsons Dis. 2016;6(4):685-698.

26. Dobkin RD, Menza M, Allen LA, et al. Cognitive-behavioral therapy for depression in Parkinson's disease: a randomized, controlled trial. Am J Psychiatry. 2011;168(10):1066-1074

27. Ellis T, Cavanaugh JT, Earhart GM, et al. Factors associated with exercise behavior in people with Parkinson disease. Phys Ther. 2011; 91(12):1838-1848.

28. Aarsland D, Marsh L, Schrag A. Neuropsychiatric symptoms in Parkinson's disease. Mov Disord. 2009;24(15):2175-2186.

29. Lorig K. Partnerships between expert patients and physicians. Lancet. 2002;359(9309):814-815

30. Lorig KR, Holman H. Self-management education: history, definition, outcomes, and mechanisms. Ann Behav Med. 2003;26(1):1-7.

31. Warsi A, Wang PS, LaValley MP, Avorn J, Solomon DH. Selfmanagement education programs in chronic disease: a systematic review and methodological critique of the literature. Arch Intern Med. 2004;164(15):1641-1649

32. Chodosh J, Morton SC, Mojica W, et al. Meta-analysis: chronic disease self-management programs for older adults. Ann Intern Med. 2005;143(6):427-438.

33. Siantz E, Aranda MP. Chronic disease self-management interventions for adults with serious mental illness: a systematic review of the literature. Gen Hosp Psychiatry. 2014;36(3):233-244.

34. Lawn S, Schoo A. Supporting self-management of chronic health conditions: common approaches. Patient Educ Couns. 2010;80(2): 205-211.

35. Lewis C, Annett LE, Davenport S, Hall AA, Lovatt P. Mood changes following social dance sessions in people with Parkinson's disease. $J$ Health Psychol. 2016;21(4):483-492.

36. Crizzle AM, Newhouse IJ. Themes associated with exercise adherence in persons with Parkinson's disease: a qualitative study. Occup Ther Health Care. 2012;26(2-3):174-186.

37. Hoehn M, Yahr MD. Parkinsonism: onset, progression and mortality. Neurology. 1967;17:427-442.

38. Montgomery SA, Asberg M. A new depression scale designed to be sensitive to change. Br J Psychiatry. 1979;134:382-389.

39. Folstein MF, Folstein SE, McHugh PR. "Mini-mental state". A practical method for grading the cognitive state of patients for the clinician. J Psychiatr Res. 1975;12(3):189-198.

40. Lawless ME, Kanuch SW, Martin S, et al. A nursing approach to selfmanagement education for individuals with mental illness and diabetes. Diabetes Spectr. 2016;29(1):24-31.

41. Sajatovic M, Dawson NV, Perzynski AT, et al. Best practices: optimizing care for people with serious mental illness and comorbid diabetes. Psychiatr Serv. 2011;62(9):1001-1003.

42. Sajatovic M, Gunzler D, Einstadter D, et al. A preliminary analysis of individuals with serious mental illness and comorbid diabetes. Arch Psychiatr Nurs. 2016;30(2):226-229.

43. Peacock CA, Sanders GJ, Wilson KA, et al. Introducing a multifaceted exercise intervention particular to older adults diagnosed with Parkinson's disease: a preliminary study. Aging Clin Exp Res. 2014;26(4):403-409.

44. Ridgel AL, Walter BL, Tatsuoka C, et al. Enhanced exercise therapy in Parkinson's disease: a comparative effectiveness trial. J Sci Med Sport. 2016;19(1):12-17.

45. Nasreddine ZS, Phillips NA, Bedirian V, et al. The Montreal Cognitive Assessment, MoCA: a brief screening tool for mild cognitive impairment. J Am Geriatr Soc. 2005;53(4):695-699.

46. Starkstein SE, Mayberg HS, Preziosi TJ, Andrezejewski P, Leiguarda R, Robinson RG. Reliability, validity, and clinical correlates of apathy in Parkinson's disease. J Neuropsychiatry Clin Neurosci. 1992;4(2): 134-139. 
47. Lipman RS. Differentiating anxiety and depression in anxiety disorders: use of rating scales. Psychopharmacol Bull. 1982;18(4):69-77.

48. Visser M, Marinus J, Bloem BR, Kisjes H, van den Berg BM, van Hilten JJ. Clinical tests for the evaluation of postural instability in patients with parkinson's disease. Arch Phys Med Rehabil. 2003; 84(11):1669-1674.

49. Goetz CG, Stebbins GT, Wolff D, et al. Testing objective measures of motor impairment in early Parkinson's disease: feasibility study of an at-home testing device. Mov Disord. 2009;24(4):551-556.

50. Piccinni A, Marazziti D, Catena M, et al. Plasma and serum brain-derived neurotrophic factor (BDNF) in depressed patients during 1 year of antidepressant treatments. J Affect Disord. 2008;105(1-3):279-283.

51. Hirsch MA, Iyer SS, Sanjak M. Exercise-induced neuroplasticity in human Parkinson's disease: what is the evidence telling us? Parkinsonism Relat Disord. 2016;22 Suppl 1:S78-S81.

52. Scalzo P, Kummer A, Cardoso F, Teixeira AL. Increased serum levels of soluble tumor necrosis factor-alpha receptor- 1 in patients with Parkinson's disease. J Neuroimmunol. 2009;216(1-2):122-125.

53. Scalzo P, Kummer A, Cardoso F, Teixeira AL. Serum levels of interleukin-6 are elevated in patients with Parkinson's disease and correlate with physical performance. Neurosci Lett. 2010;468(1):56-58.

54. Pedersen BK, Bruunsgaard H, Ostrowski K, et al. Cytokines in aging and exercise. Int J Sports Med. 2000;21 Suppl 1:S4-S9.

55. Pedersen BK, Hoffman-Goetz L. Exercise and the immune system regulation, integration, and adaptation. Physiol Rev. 2000;80(3): 1055-1081.
56. Tomlinson CL, Stowe R, Patel S, Rick C, Gray R, Clarke CE. Systematic review of levodopa dose equivalency reporting in Parkinson's disease. Mov Disord. 2010;25(15):2649-2653.

57. Chaudhry S, Jin L, Meltzer D. Use of a self-report-generated Charlson Comorbidity Index for predicting mortality. Med Care. 2005;43(6): $607-615$.

58. Poewe W. Dysautonomia and cognitive dysfunction in Parkinson's disease. Mov Disord. 2007;22 Suppl 17:S374-S378.

59. Ishihara L, Brayne C. A systematic review of depression and mental illness preceding Parkinson's disease. Acta Neurol Scand. 2006; 113(4):211-220.

60. Pedersen KF, Larsen JP, Tysnes OB, Alves G. Prognosis of mild cognitive impairment in early Parkinson disease: the Norwegian ParkWest study. JAMA Neurol. 2013;70(5):580-586.

61. Nguyen TT, Glass L, Coles CD, et al. The clinical utility and specificity of parent report of executive function among children with prenatal alcohol exposure. J Int Neuropsychol Soc. 2014;20(7):704-716.

62. Scalzo P, Kummer A, Bretas TL, Cardoso F, Teixeira AL. Serum levels of brain-derived neurotrophic factor correlate with motor impairment in Parkinson's disease. J Neurol. 2010;257(4):540-545.

63. Frazzitta G, Maestri R, Ghilardi MF, et al. Intensive rehabilitation increases BDNF serum levels in parkinsonian patients: a randomized study. Neurorehabil Neural Repair. 2014;28(2):163-168.

64. Winter B, Breitenstein C, Mooren FC, et al. High impact running improves learning. Neurobiol Learn Mem. 2007;87(4):597-609.
Patient Preference and Adherence

\section{Publish your work in this journal}

Patient Preference and Adherence is an international, peer-reviewed, open access journal that focuses on the growing importance of patient preference and adherence throughout the therapeutic continuum. Patient satisfaction, acceptability, quality of life, compliance, persistence and their role in developing new therapeutic modalities and compounds to optimize

\section{Dovepress}

clinical outcomes for existing disease states are major areas of interest for the journal. This journal has been accepted for indexing on PubMed Central. The manuscript management system is completely online and includes a very quick and fair peer-review system, which is all easy to use. Visit http://www. dovepress.com/testimonials.php to read real quotes from published authors. 